Proceedings of the ASME 2020 39th International Conference on Ocean, Offshore and Arctic Engineering

OMAE2020

August 3-7, 2020, Virtual, Online

OMAE2020-18982

\title{
DESIGN AND TESTING OF AUV DOCKING MODULES FOR A RENEWABLY POWERED OFFSHORE AUV SERVICING PLATFORM
}

\author{
Maha N. Haji* \\ Department of Aeronautics and Astronautics \\ Massachusetts Institute of Technology \\ Cambridge, Massachusetts, 02139 \\ and \\ Sibley School of Mechanical and Aerospace Engineering \\ Cornell University \\ Ithaca, New York, 14853 \\ Email: mhaji@mit.edu

\section{Johannes Norheim \\ Olivier L. de Weck} \\ Department of Aeronautics and Astronautics \\ Massachusetts Institute of Technology \\ Cambridge, Massachusetts, 02139
}

\author{
Jimmy Tran \\ Department of Mechanical Engineering \\ Massachusetts Institute of Technology \\ Cambridge, Massachusetts, 02139
}

\begin{abstract}
Autonomous Underwater Vehicle (AUV) missions are limited in range and duration by the vehicle's battery capacity, and sensor payloads are limited by the processing power onboard which is also restricted by the vehicle's battery capacity. Furthermore, the power consumption of a vehicle's acoustic system limits the possibility of substantial data transmission, requiring the AUV be retrieved to download most data. The Platform for Expanding AUV exploRation to Longer ranges (PEARL), described in this paper, aims to extend the range and endurance of AUVs while reducing data latency and operating costs. PEARL is an integrated autonomous floating servicing station that utilizes renewable energy to simultaneously provide AUV battery recharging and data uplink via new generation high-bandwidth low-Earth orbit satel-
\end{abstract}

\footnotetext{
*Address all correspondence to this author.
}

lite constellations. This paper details the design and testing of two potential AUV docking modules of the PEARL system. The modules are uniquely located near the ocean surface, an energetic environment that presents a particular set of challenges for $A U V$ docking. The results will be used to inform the design of a prototype system to be tested in an ocean setting.

\section{INTRODUCTION}

Autonomous Underwater Vehicles (AUVs) offer the ability to provide persistent and expanded ocean observations and measurements. The battery capacity of AUVs, however, currently limits the range and duration of missions. At the same time, sensor payload and ocean measurement resolution are limited by the processing power onboard AUVs, which is also restricted by 
the vehicle's battery capacity. Autonomy in underwater vehicles is poised to be the next technological push in the industry, however this autonomy requires real-time data analysis which relies on processing power. AUV range and endurance become severely limited as the power consumption of onboard processors approaches that of the propulsion system. Furthermore, the power consumption of a vehicle's acoustic system limits the possibility of substantial data transmission. These limitations require that AUVs be frequently recovered to recharge and offload data, a process which generally requires the assistance of a support vessel and crew, which can cost in excess of $\$ 30,000$ per day $[1,2]$.

The Platform for Expanding AUV exploRation to Longer ranges (PEARL) aims to remove these limitations and reduce AUV operating costs by providing an integrated autonomous floating docking station which simultaneously provides AUV battery recharging and data uplink via the new generation of high-bandwidth low-Earth orbit (LEO) constellations (OneWeb, Starlink, Iridium NEXT) [3]. This ensures worldwide connectivity and control of AUVs, allowing for near-real-time underwater data from across the globe. By utilizing solar power paired with integrated battery modules, PEARL can harvest power during daytime hours, allowing reliable, on-demand recharging of vehicles and data transfer, effectively extending AUV endurance and return. Fig. 1 shows an example use-case for PEARL in the open ocean.

Past docking, power, and data transfer platforms for AUVs were limited in mobility, power generation, and range of vehicles serviceable. With a recharging system predicted to save $35 \%$ on AUV operating costs [4], PEARL seeks to overcome some of the

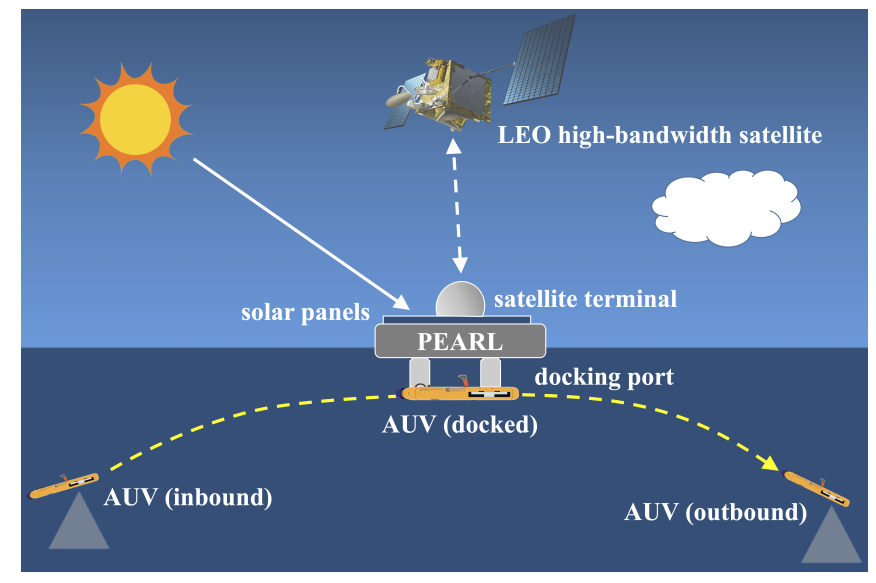

FIGURE 1. AN INBOUND AUV CAN DOCK TO PEARL, WHICH SIMULTANEOUSLY RECHARGES THE VEHICLE USING RENEWABLE SOLAR POWER, AND OFFLOADS DATA VIA A HIGH-BANDWIDTH LEO SATELLITE LINK, ENABLING THE AUV TO CONDUCT LONGER-RANGE MISSIONS AND COLLECT HIGHER RESOLUTION OCEAN MEASUREMENTS. past limitations in AUV service platforms by co-optimizing the design and operation of underwater, surface, and space assets.

The design of PEARL consists of an upper floating platform and a lower damping plate, connected by a structural spar. The floating platform houses solar panels and a satellite terminal. Furthermore, the floating platform could be shaped like a hexagon, which may enable multiple servicing platforms to connect together for increased power and data transfer capabilities. The submerged damping plate reduces heave motion of the platform and is a promising location for the possible docking modules.
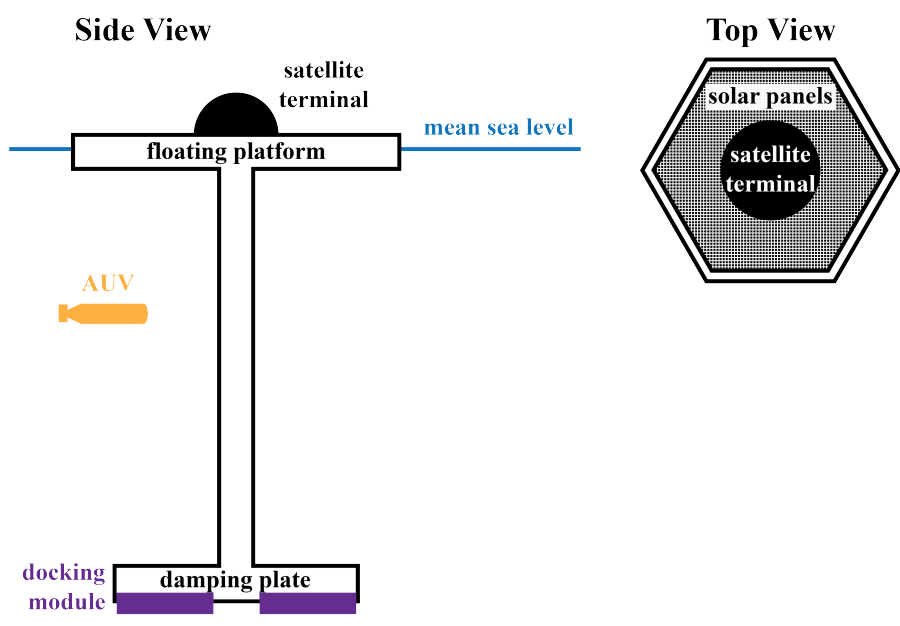

FIGURE 2. SCHEMATIC OF THE PLATFORM FOR EXPANDING AUV EXPLORATION TO LONGER RANGES (PEARL).

The main challenge in the development of PEARL is the coupling of many parameters. The number of underwater docking ports and servicing frequency of AUVs drives the dimensions, ports, and mass distribution of the platform. The size and efficiency of the solar panels determines the amount of power that can be collected and stored onboard. The battery chemistry dictates the recharge rates of AUVs, and number of recharge cycles to be supported. Finally, the hydrodynamics and stability of the platform determines the antenna pointing and link budget of the AUV-platform-to-satellite link. Design of such a platform is a complex problem well suited to multidisciplinary design optimization (MDO) with many parameters and interdisciplinary links between structures, robotics, power management, hydrodynamics, and satellite communications. MDO methodology is currently being employed in the design of PEARL with the parameters of the AUV and satellite constellation taken as a given and the design of the servicing platform being optimized for minimizing the AUV operating cost $(\$ / \mathrm{hr})$. 


\section{AUV DOCKING MODULE}

One of the key challenges in the development of PEARL is the ability of the AUV to reliably dock and undock from the platform. AUV docking stations can traditionally be classified into either directional funnel docks [5-8] or omnidirectional pole docks [9]. In the former, the AUV homes into a funnel and is guided in by bouncing off and sliding along the funnel face. Funnel docks have a large capture envelope and can be constructed to fully constrain the AUV after capture. However, the funnel dock is fundamentally directional in nature and therefore imposes constraints on the approach direction, requiring the AUV to maneuver with awareness of the docking orientation, which can be difficult in the presence of currents.

Pole docks, on the other hand, are vertical structures such as rigid poles or cables under tension, to which the AUV attaches with some latching. Unlike funnel docks, pole docks have a large vertical capture area and only a relatively small horizontal capture area, and allow an AUV to approach from any direction. This eliminates the need to control the dock orientation in the horizontal plane or for the dock to communicate the orientation to the AUV, enabling the AUV to approach into the current. However, the use of pole docks requires the AUV to carry some sort of mechanism for attaching itself to the pole.

In the design of docking modules for PEARL, the following functional requirements were considered:

1. The dock must be omnidirectional with $360^{\circ}$ full accessibility.

2. The dock must be lightweight with a total mass at or below $50 \mathrm{~kg}$.

3. The dock must be able to adapt to a wide range of AUV sizes from a diameter of 0.1 to $0.3 \mathrm{~m}$ and a length of 0.5 to $3.0 \mathrm{~m}$.

4. The dock must operate for at least 720 hours (one month) without cleaning in the presence of biofouling.

5. The probability of successful docking for any given attempt must be at least $90 \%$.

Two docking module designs seeking to meet the above functional requirements are detailed in this paper. The first utilizes a pair of hooks on the AUV that allow it to catch onto the dock and pivot until it mates with a docking connector on the damping plate of PEARL. The second uses a pair of whiskers on the nose cone of the AUV that allow it to grab onto ropes strung between the upper float and lower damping plate of PEARL. For the purposes of this study, the AUV to be modified for use with PEARL is considered to be the Bluefin-9 developed by General Dynamics Mission Systems.

\section{HOOK DOCKING DESIGN}

The hook docking system utilizes similar principles as the Marine Bird underwater docking base developed by Kawasaki et al. $[10,11]$. In the case of the Marine Bird, the docking concept took inspiration from the landing of an aircraft onto an aircraft carrier in that the AUV runs slowly over the docking base until it catches a V-shaped guide on the base using the AUV's catching arm. The vehicle then continues moving forward until it joins with a connecting device on the base that provides inductive recharging.

For the hook docking system presented here, the AUV is outfitted with a pair of buoyant hooks on a flexible line that are deployed from a winching system within the vehicle when it enters docking mode, as shown in Fig. 3 for the case of a Bluefin-9. The docking module on PEARL is located on the underside of the damping plate below the surface of the water to reduce waveinduced motions. Docking the AUV on the damping plate also has the added benefit of decreasing the overall motions of the PEARL platform by lowering the center of mass due to the additional mass joining the bottom of the structure.

When entering docking mode, the buoyant hooks are released from the AUV body and float upward. As the AUV approaches the docking module, its hooks snag onto a docking ring on the PEARL system, as shown in Fig. 4. The docking ring has a series of capture points that the hooks slide into, guiding the AUV into alignment. From this position, as the AUV continues to propel forward, it begins to translate upward until a socket on the top of the AUV (shown in green) clicks in place with a ball joint in the center of the docking ring (shown in green), as depicted in Fig. 5. This ball-and-socket connector allows for inductive recharging of the AUV, while ensuring the AUV maintains close proximity to PEARL to allow for straightforward acoustic data transfer, with PEARL either storing the data locally for satellite transmission at a later time, or transmitting the data directly to a satellite constellation. One issue, however, is the fact that the transfer of substantial amounts of data using the acoustic system onboard the vehicle could consume considerable amounts of power (since AUV acoustic systems are

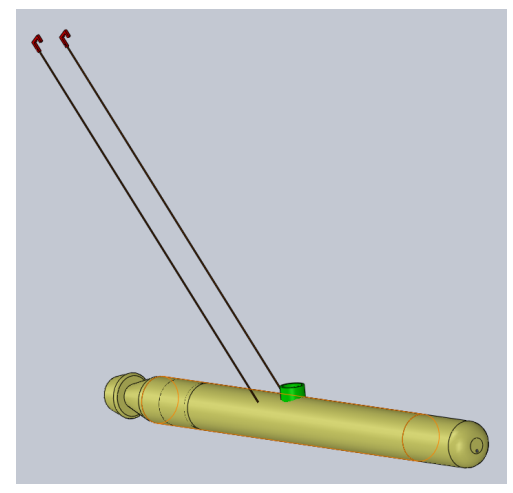

FIGURE 3. 3D MODEL OF AUV MODIFIED WITH DEPLOYABLE HOOKS FOR AUTONOMOUS DOCKING TO PEARL.HOOKS SHOWN IN DEPLOYED STATE. 


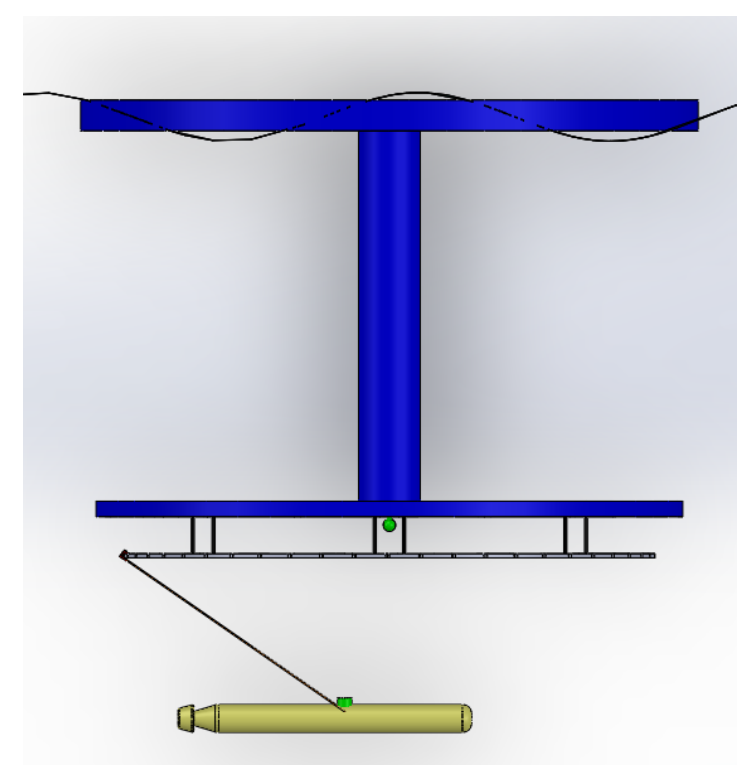

FIGURE 4. AUV IN DOCKING STATE WITH HOOKS LATCHING ON TO A DOCKING RING ATTACHED TO THE DAMPING PLATE OF PEARL.

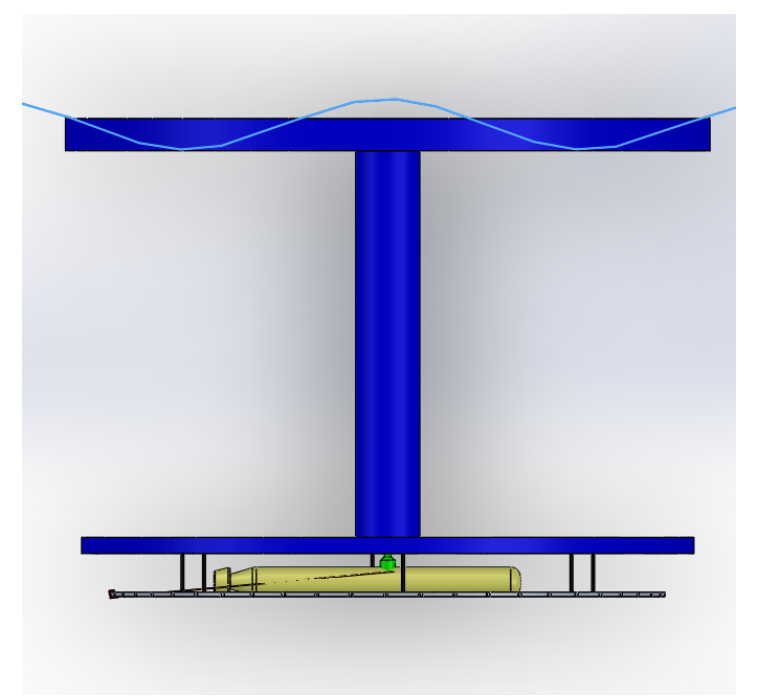

FIGURE 5. AUV IN DOCKED STATE WITH DOCKING SOCKET ON AUV SECURELY CONNECTED TO DOCKING BALL ON PEARL DAMPING PLATE.

usually optimized for low-data-rate and longer-range capabilities for long baseline navigation and limited communications), possibly negating the hours of vehicle battery recharging provided by PEARL. Therefore, data transfer via an uplink connecter embedded in the ball-and-socket connector should be investigated.

In order to disengage from the dock, the AUV actuates a threaded bolt out from its socket connector to effectively push the socket off of the ball joint in PEARL. This releases the AUV and allows it to maneuver in reverse to remove the hooks from the docking ring. The vehicle then winches up the cable and hooks into its body and resumes its mission.

\section{Docking Dynamics}

In docking mode, the hook connects the AUV to the docking ring on the servicing platform, thereby imparting a tension force on the AUV, as shown in Fig. 6.

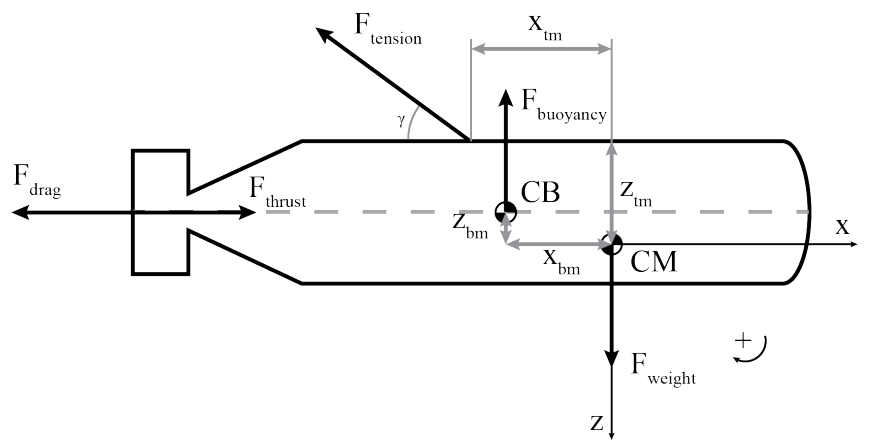

FIGURE 6. AUV LONGITUDINAL FREE BODY DIAGRAM ONCE HOOKS ARE CONNECTED TO DOCKING RING ON PEARL.

This alters the AUV equations of motion to become:

$$
\begin{gathered}
\sum F_{\text {ext }, x}=F_{\text {thrust }}-F_{\text {drag }}-F_{\text {tension }} \cos (\gamma)=m \ddot{x} \\
\sum F_{\text {ext }, z}=F_{\text {buoyancy }}-F_{\text {weight }}+F_{\text {tension }} \sin (\gamma)=m \ddot{z}
\end{gathered}
$$

$$
\begin{aligned}
\sum M_{\text {ext }, C M}= & F_{\text {buoyancy }} x_{\text {bm }}+F_{\text {tension }} \sin (\gamma) x_{\text {tm }}+F_{\text {thrust }} z_{b m} \\
& -F_{\text {tension }} \cos (\gamma) z_{\text {tm }}-F_{\text {drag }} z_{b m}=I \ddot{\theta}
\end{aligned}
$$

where

$x$ is the displacement in the $x$ direction of the AUV, $z$ is the displacement in the $z$ direction of the AUV, $\theta$ is the angular displacement of the AUV,

$C B$ is the AUV center of buoyancy,

$C M$ is the AUV center of mass and the origin of the AUV body-coordinate system, 
$\gamma$ is the angle the hooks make with respect to the AUV body, $F_{\text {thrust }}$ is the force of the AUV propulsion,

$F_{\text {drag }}$ is the drag force on the AUV body,

$F_{\text {tension }}$ is the tension force in the cable connecting the AUV to the hooks,

$F_{\text {buoyancy }}$ is the buoyant force of the AUV,

$F_{\text {weight }}$ is the weight of the AUV,

$x_{b m}$ is the distance in the $x$ direction from the AUV's center of mass to its center of buoyancy,

$z_{b m}$ is the distance in the $z$ direction from the AUV's center of mass to its center of buoyancy,

$x_{t m}$ is the distance in the $x$ direction from the attachment point of the hook cable on the AUV to its center of mass, and

$z_{t m}$ is the distance in the $z$ direction from the attachment point of the hook cable on the AUV to its center of mass.

As can be seen from Eqn. 3, given that AUVs are accustomed to maneuvering in the presence of the forces of drag, thrust, buoyancy, and weight, the addition of the tension force from the hooked cables will likely result in a pitching moment. The AUV will thus pitch as it traverses upwards towards the docking connector and this motion will need to be accounted for in the vehicle's docking programming.

\section{Docking Connector}

An additional key element in the design of the hook docking system is the ball-and-socket joint that connects the AUV to the servicing platform. An example of such a joint is shown in Fig. 7. The amount of force required to connect the socket and ball as well as the amount of force to disengage the socket from the ball in this design is the topic of current research. (a)

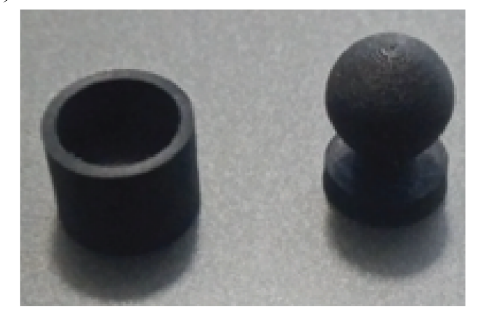

(b)

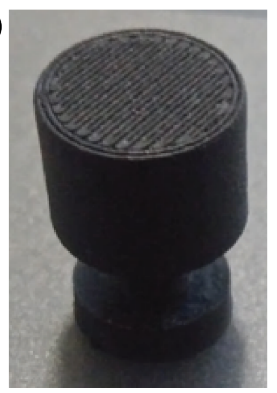

FIGURE 7. BALL-AND-SOCKET JOINTS TO BE USED AS DOCKING CONNECTORS IN THE HOOK DOCKING DESIGN. (A) SHOWS THE SOCKET AND BALL SEPARATELY AND (B) SHOWS THEM CONNECTED.

\section{Design Considerations}

A number of elements must be considered in the design of the hook docking concept. Firstly, aligning the AUV with the ball-and-socket docking connector may be very difficult in an ocean setting. Secondly, in order for the hooks to stay at the vehicle center, the AUV will either need a wet payload or be able to handle extra vehicle drag when the hooks are not deployed. Furthermore, for the hooks to stay above the vehicle when the AUV is in motion the hooks must be extremely buoyant. Finally, the hooks will likely need to be on a rigid cable so as to prevent tangling between the two cables and tangling between the cables and the propeller.

\section{WHISKER DOCKING DESIGN}

The whisker docking system takes inspiration from the Line Capture, Line Recovery (LCLR) technology developed by [12] for the REMUS 100. Piskura et al. modified the REMUS 100 AUV with a line capture cone on the nose of the AUV consisting of a V-Wing depressor [12]. In this work, we consider the case if a similar such nose cone were developed for an AUV in order to dock autonomously with the PEARL system.

Figs. 8 and 9 show a 3D model of what the modified nose of the Bluefin-9 AUV might look like with the addition of a set of "whiskers" that can grab onto a rope. In Fig. 9 the whisker arms are shown in green and an additional latching mechanism is shown in red.

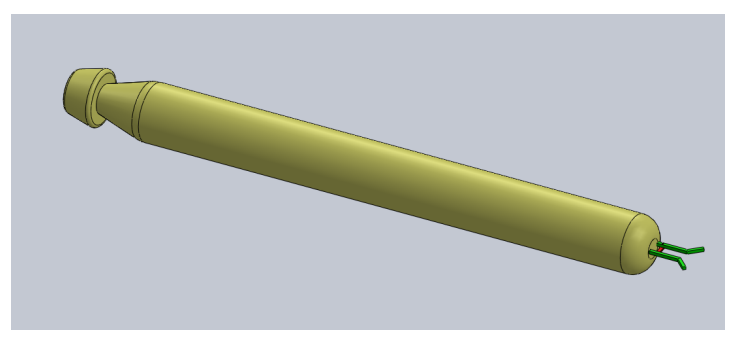

FIGURE 8. AUV MODIFIED WITH DEPLOYABLE WHISKERS FOR AUTONOMOUS DOCKING TO PEARL. WHISKERS SHOWN IN DEPLOYED STATE.

When entering the docking sequence, the AUV releases a pair of whiskers stored on the sides of its nose cone. It then approaches the PEARL system which is outfitted with a series of ropes between the upper float and lower damping disk for the AUV to dock to. In the docked position, shown in Figs. 10 and 11 , the latch mechanism closes around the rope to keep the rope within the grasps of the whiskers. The ropes could be embedded with electronics to allow for inductive recharging of the AUV. Additionally, the proximity of the AUV with PEARL allows for 


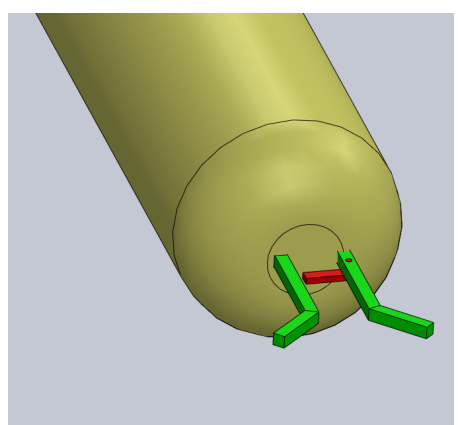

FIGURE 9. CLOSE-UP OF WHISKERS ON AUV. WHISKERS ARE SHOWN IN GREEN WITH AN ADDITIONAL LATCHING MECHANISM SHOWN IN RED. IN DOCKED STATE, LATCHING MECHANISM WILL CLOSE AROUND ROPE TO ENSURE AUV MAINTAINS POSITION.

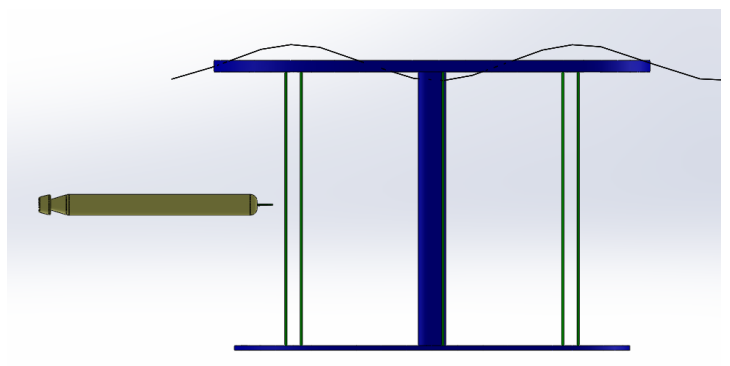

FIGURE 10. AUV IN DOCKING STATE WITH WHISKERS DEPLOYED APPROACHING PEARL. ROPES BETWEEN THE UPPER FLOAT AND LOWER DAMPING PLATE ON PEARL SERVE AS ANCHOR POINTS FOR THE AUV.

acoustic data transfer to PEARL. However, as in the hook docking design, acoustic data transfer could consume more power than is provided to the AUV via recharging. Thus, in the case of the whisker docking system, data transfer via the inductive charging cables should be investigated.

To disengage from the dock, the AUV releases the latching mechanism to free it from the rope and reverses back from PEARL. Once it is a safe distance away from the platform, it retracts its whiskers and resumes its mission.

\section{Docking Dynamics}

Similar to many previously proposed pole-docking systems, in order to dock with the rope the AUV must propel itself to the rope and intercept the rope at speed. Therefore, in the design of the whisker docking system one critical design parameter is the diameter of the docking ropes to ensure they can handle the tensile stress induced by the AUV as it collides with them to dock.

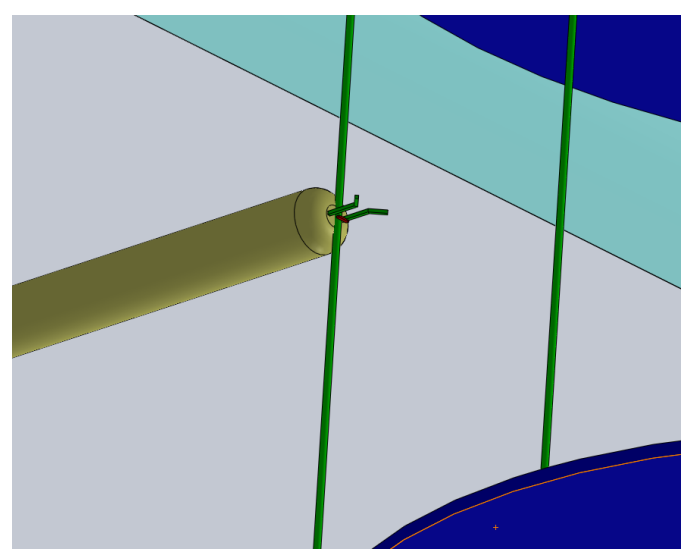

FIGURE 11. CLOSE-UP OF AUV IN DOCKED POSITION. WHISKERS IN GREEN GUIDE THE ROPE TO BE SECURED BY THE AUV USING THE LATCH MECHANISM, SHOWN IN RED.

Assuming a constant velocity, the equation of motion for the AUV moving forward is

$$
F_{\text {thrust }}-F_{\text {drag }}=0
$$

where $F_{\text {thrust }}$ and $F_{\text {drag }}$ are as defined previously in the case of the hook docking design. Thus, the thrust force will be equal to the force of drag, which is given by

$$
F_{\text {drag }}=\frac{1}{2} C_{D} \rho v^{2} A
$$

where

$C_{d}$ is the AUV's drag coefficient,

$\rho$ is the density of seawater,

$v$ is the velocity of the AUV, and

$A$ is the cross-sectional area of the AUV.

In the case of the Bluefin-9, the AUV considered for this study, the AUV has a diameter of $0.23 \mathrm{~m}$, resulting in a crosssectional area of $A=0.04154 \mathrm{~m}^{2}$. Taking the drag coefficient to be $C_{d}=0.1$, a reasonable value for torpedo-shaped AUVs, and the speed of approach to be the AUV's maximum transit speed, $v=3 \mathrm{~m} / \mathrm{s}$, the resulting drag force is $F_{d r a g}=19.2 \mathrm{~N}$, an extremely small value.

Another method to determine the force the rope must withstand can be done by considering the impulse imparted to the rope when the AUV intercepts it, as

$$
F_{\text {collide }}=\frac{m \Delta v}{\Delta t}
$$


In the case of the Bluefin-9, the AUV mass is $m=70 \mathrm{~kg}$. Considering the AUV slows to rest from its maximum transit speed of $3 \mathrm{~m} / \mathrm{s}$ within the span of $0.01 \mathrm{~s}$, the resulting collision force is $F_{\text {collide }}=21,000 \mathrm{~N} \approx 4,720 \mathrm{lbf}$. Including a safety factor of two, the docking rope must withstand a force of approximately 9,400 lbf (42,000 N). For existing Amsteel Blue high-strength mooring lines, this corresponds to a rope diameter of 5/16" which has a tensile strength of $13,700 \mathrm{lbf}(\approx 60,941 \mathrm{~N})[13]$.

\section{Design Considerations}

As with the hook docking concept, there are a number of considerations to be taken into account in developing the whisker docking design. Firstly, the docking system may need to maintain tension in a rope that spans the upper platform and lower damping plate of PEARL, which may be difficult.

Secondly, due to the fact that most AUVs are ballasted to maintain $1 \%$ net positive buoyancy unless they have a variable ballast system, once latched to the line if there is no stop the vehicle will continue to float upward along the line until it meets the platform's upper float. Presently, variable ballast systems are complicated and unreliable and are therefore not of widespread use in most vehicles. With a stop on the line implemented, the vehicle will float up until it reaches a system equilibrium. Since docking occurs at the forward end of the vehicle and the center of buoyancy is at the middle of the vehicle, this one half vehicle length lever arm for most vehicles results in an approximately $65^{\circ}$ pitch down to achieve system equilibrium. This pitch angle will need to be considered in the design of the whiskers on the AUV and rope on PEARL to ensure sufficient connection for inductive charging and embedded data transfer.

\section{DOCKING MODULE TESTING}

Research is underway to determine the optimal design of PEARL, which will include details regarding the floating platform size, the damping plate size and depth below the ocean surface, and the system's battery requirements (which will likely be the main driver of the platform's mass).

Also in progress is pool testing of the hook and whisker docking modules to determine the feasibility of each concept for integration with PEARL. Some key concerns include the ability for an AUV to dock with a platform moving under the influence of waves, the force requirements for docking, and the force and maneuvering requirements for undocking.

\section{Model scaling}

Specifically, Froude-scaled models of the two different docking modules detailed in this proposal were fabricated for testing. The geometric scale used by the models is given by $\lambda=\frac{L_{f}}{L_{m}}$, where $L_{f}$ is the full-scale characteristic length and $L_{m}$ is the model scale characteristic length. Matching the Froude
TABLE 1. SOME KEY DIMENSIONS OF THE AUV (BLUEFIN-9) AT FULL AND 1:5 FROUDE-SCALED MODEL.

\begin{tabular}{llll}
\hline AUV Parameter & Unit & Full-Scale & $\mathbf{1 : 5}$ Froude-scaled \\
\hline Diameter & $\mathrm{m}$ & 0.23 & 0.046 \\
Length & $\mathrm{m}$ & 2.5 & 0.625 \\
Mass & $\mathrm{kg}$ & 70 & 0.56 \\
\hline
\end{tabular}

TABLE 2. SOME KEY DIMENSIONS OF PEARL AT FULL AND 1:5 FROUDE-SCALED MODEL.

\begin{tabular}{llll}
\hline PEARL Parameter & Unit & Full-Scale & 1:5 Froude-scaled \\
\hline Diameter & $\mathrm{m}$ & 3.75 & 0.75 \\
Draft & $\mathrm{m}$ & 2.1 & 0.42 \\
Mass & $\mathrm{kg}$ & 3500 & 28 \\
\hline
\end{tabular}

number between the full and model scale using this geometric scale results in different scale ratios for various physical parameters. For instance, while acceleration varies only by a ratio of 1 between the model and full scale, the force scales by a factor of $\lambda^{3}$ [14]. The geometric scale used in fabricating the docking concepts was $\lambda=5$, resulting in 1:5 Froude-scale models.

One key piece in the testing of the docking modules is the AUV. To model the AUV, a remote-controlled vehicle stand-in was fabricated at a 1:5 Froude scale. Table 1 details the key dimensions of the AUV stand-in. The ability to easily control the AUV stand-in via a remote will be key to testing out docking at varying speeds and in a variety of wave environments. Similarly, Table 2 outlines some of the key dimensions of the PEARL prototype fabricated for pool testing.

\section{Initial pool test results}

Initial pool tests of the PEARL platform and whisker docking module were conducted. The fabricated AUV stand-in used for the tests is shown in Fig. 12 and the fabricated PEARL prototype is shown in Fig. 13.

Motion tracking of the AUV stand-in and the PEARL prototype using MATLAB's Computer Vision Toolbox was used to determine approximate measurements of relative motion when the whiskers on the vehicle collide with the rope to successfully dock with PEARL. Fig. 14 shows an example of the average velocity determined for a number of points on the AUV stand-in for one successful docking test. Initial analysis showed that the impact of the AUV stand-in on the PEARL prototype induced motion on the platform, suggesting that stationkeeping should be 


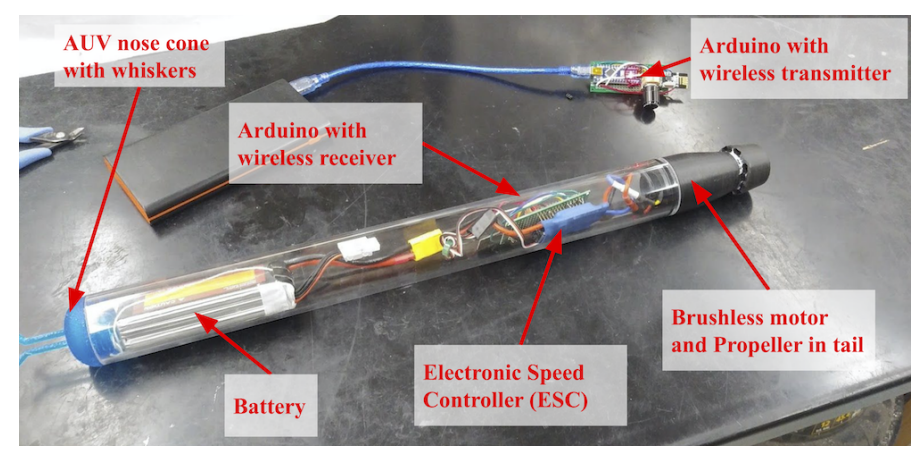

FIGURE 12. REMOTE-CONTROLLED "AUV STAND-IN" FABRICATED AT 1:5 FROUDE-SCALED AND USED IN POOL TESTING.
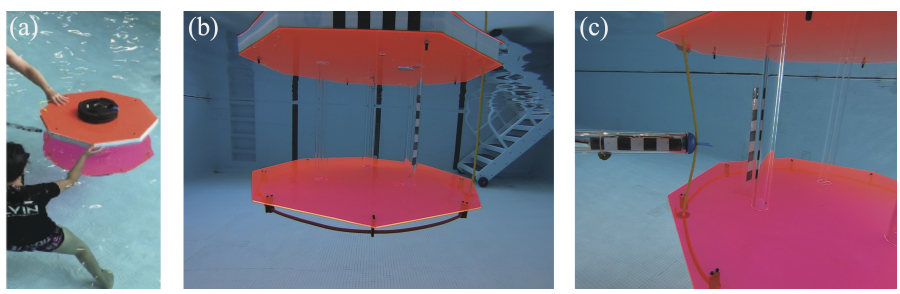

FIGURE 13. PHOTOGRAPHS OF POOL TESTING OF 1:5TH FROUDE-SCALED PEARL PROTOTYPE FROM (A) ABOVE THE WATER, (B) UNDER THE WATER, AND (C) SHOWING A SUCCESSFUL DOCKING OF THE AUV STAND-IN USING THE WHISKER DOCKING CONCEPT.

considered in future designs of PEARL. However, the fact that the motion upon impact of PEARL was generally 1-2 orders of magnitude less than the motion of the AUV indicates that the initial designs of the platform may be robust to the AUV docking force. These results will be investigated further with controlled tow tank testing of prototypes of the AUV stand-in and PEARL.

\section{CONCLUSIONS AND FUTURE WORK}

This paper detailed two design concepts for the docking module of a Platform for Expanding AUV exploRation to Longer ranges (PEARL). PEARL is an integrated autonomous floating servicing station that utilizes renewable energy to simultaneously provide AUV battery recharging and data uplink via the new generation of high-bandwidth low-Earth orbit satellite constellations. One key component in the design of PEARL is the docking module to connect incoming AUVs with the servicing platform to allow for AUV recharging and data offloading.

One docking concept utilizes a pair of hooks on the AUV that allow it to catch onto the dock and pivot until it mates with a docking connector on the damping plate of PEARL. The second concept uses a pair of whiskers on the nose cone of the AUV that allow it to grab onto ropes strung between the upper float

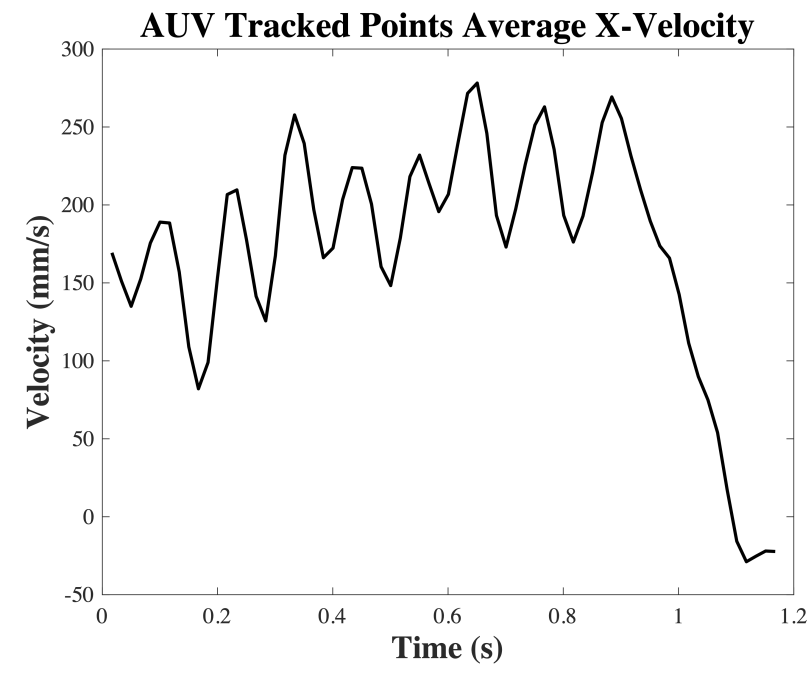

FIGURE 14. AVERAGE VELOCITY OF POINTS TRACKED ON AUV STAND-IN DURING ONE SUCCESSFUL TEST OF THE WHISKER DOCKING CONCEPT. THE SHARP CHANGE IN VELOCITY FROM 0.9-1.1 SECONDS IS THE POINT AT WHICH THE WHISKERS ON THE VEHICLE COLLIDED WITH THE ROPE ON PEARL.

and lower damping plate of PEARL. Continued work entails further pool testing of the 1:5 Froude-scaled prototypes of each design concept in order to determine their feasibility for use with PEARL.

The results from the MDO studies will inform the design of PEARL fabricated for tow tank testing to determine the structure's response to ocean waves. The tow tank tests will also allow for a better understanding of the challenges for autonomously docking AUVs in an energetic ocean environment due to the unique location of the docking modules near the ocean surface. The results will be used to inform the design of a prototype system to be tested in an ocean setting planned for summer 2020.

\section{ACKNOWLEDGMENT}

Thank you to the MIT Portugal Program for the seed funding to conduct this research. Thank you to Dr. James Bellingham and Prof. Hanumant Singh for their guidance in understanding the challenges and previous work related to AUV docking at sea. Thank you as well to Judson Poole for providing his insights on our paper.

\section{REFERENCES}

[1] Monterey Bay Aquarium Research Institute. MBARIRates for vessels, vehicles, MARS, labor, test tank. URL http://www.mbari.org/at-sea/mars-ship-rates/. 
[2] Podder, T., Sibenac, M., and Bellingham, J., 2004. "AUV docking system for sustainable science missions". In IEEE Int. Conf. Robot. Automat., Vol. 5, pp. 4478-4484.

[3] Foreman, V. L., Siddiqi, A., and de Weck, O., 2017. "Large satellite constellations: case studies of OneWeb and SpaceX proposals". AIAA SPACE and Astronautics Forum, p. 5200.

[4] Jr, M. A. E., 2014. "Design of an autonomous underwater vehicle (AUV) charging system for underway". Phd thesis, Massachusetts Institute of Technology.

[5] Brighenti, A., Zugno, L., Mattiuzzo, F., and Sperandio, A., 1998. "Eurodocker- a universal docking-downloading recharging system for AUVs: Conceptual design results". In Proc. of MTS/IEEE OCEANS Conference, Vol. 3, pp. 1463-1467.

[6] Knepper, S., Niemeyer, M., R. Galletti, A. B., Bjerrum, A., and Andersen, N., 2001. "Eurodocker-a universal dockingdownloading-recharging system for AUVs". WIT Trans. Built Environ, 53, pp. 1-9.

[7] McEwen, R. S., Hobson, B. W., McBride, L., and Bellingham, J. G., 2008. "Docking control system for a 54-cmdiameter (21-in) AUV". IEEE J. Ocean. Eng., 33, pp. 550562.

[8] Stokley, R., Allen, B., Austin, T., Goldsborough, R., Forrester, N., Purcell, M., and von Alt, C., 2001. "Enabling technologies for remus docking: An integral component of an autonomous ocean-sampling network". IEEE J. Ocean Eng., 26(4), pp. 487-497.

[9] Singh, H., Bellingham, J. G., Hover, F., Lemer, S., Moran, B. A., von der Heydt, K., and Yoerger, D., 2001. "Docking for an autonomous ocean sampling network". IEEE J. Ocean Eng., 26(4), pp. 498-514.

[10] Fukasawa, T., Noguchi, T., Kawasaki, T., and Baino, M., 2003. "'MARINE BIRD", a new experimental AUV with underwater docking and recharging system". In Oceans 2003. Celebrating the Past ... Teaming Toward the Future (IEEE Cat. No.03CH37492), pp. 2195-2200.

[11] Kawasaki, T., Fukasawa, T., Noguchi, T., and Baino, M., 2003. "Development of AUV "Marine Bird" with underwater docking and recharging system". In 2003 International Conference Physics and Control. Proceedings (Cat. No.03EX708), pp. 166-170.

[12] Piskura, J. C., Purcell, M., Stokey, R., Austin, T. C., Tebo, D., Christensen, R., and Jaffre, F., 2016. "Development of a robust Line Capture, Line Recovery (LCLR) technology for autonomous docking of AUVs". OCEANS 2016 MTS/IEEE Monterey, pp. 1-5.

[13] R \& W Rope. Amsteel Blue -12 Strand Dyneema Rope. URL https://rwrope.com/products /amsteel-blue-12-stranddyneema-rope?variant=29561339707445.

[14] Chakrabarti, S. K., 1994. Offshore Structure Modeling. World Scientific, Singapore, SGP. 